\title{
CORRECTIONS TO MY PAPER “ON THE STRUCTURE OF COMPLETE LOCAL RINGS"1)
}

\author{
MASAYOSHI NAGATA
}

The proof of Proposition 2 and that of Corollary to Proposition 3 in my previous paper "On the structure of complete local rings"1) are not correct." Here we want to correct them.

\section{Proof of Proposition 2.}

Since the previous proof of Proposition 2 is valid when $R / \mathrm{m}$ is perfect, we treat only the case when $R_{/}^{\prime} \mathrm{m}$ is not perfect.

Starting from $K_{0}=R / \mathrm{m}$, we obtain $K_{n}(n=1,2, \ldots)$ from $K_{n-1}$ by adjoining all $p$-th roots of elements of $K_{n-1}$.

Definition. Let a local ring $R_{1}$ with maximal ideal $m_{1}$ be a subring of another local ring $R_{2}$ with maximal ideal $\mathrm{m}_{2}$. We say that $R_{2}$ is unramified with respect to $R_{1}$ if $\mathrm{m}_{2}=\mathrm{m}_{1} R_{2}$ and $\mathrm{m}_{2}^{k} \cap R_{1}=\mathrm{m}_{1}^{k}$ for every positive integer $k$.

(1) Equal characteristic case.

We construct a sequence of local rings $R=R^{(0)} \subset R^{(1)} \subset$.. such that (1) $R^{(n)}$ is unramified with respect to $R,(2) R^{(n)} / \mathrm{m} R^{(n)}=K_{n}$ and $(3)\left(R^{(n)}\right)^{p} \subseteq R^{(n-1}$.

The existence of such a sequence obviously follows from Zorn's Lemma if we observe that a monic polynomial $f(x)$ over a local ring, say $R^{*}$, is irreducible mcdulo its maximal ideal, then $R^{*}[x] /(f(x))$ is unramified with respect to $R^{\prime}$. (We may use the $p$-basis).

Let $S$ be the union of all $R^{(n)}$. Then $S$ is a local ring unramified with respect to $R$. For every element $a^{*}$ of $R / \mathrm{m}$. we construct a sequence $\left(a_{n}\right)$ as follows: Let $b_{n}$ be a representative of $a^{* p^{-1}}$ in $R_{n}$ and set $a_{n}=b_{n}^{p^{n}}$. Then $a_{n} \in R$ and the limit $a$, which is the multiplicative representative of $a^{*}$, is in $R$. Thus we have Proposition 2 in this case.

(2) Unequal characteristic case.

As in above, we construct a sequence of local rings $R=R^{(0)} \subset R^{(1)} \subset \ldots$ satisfying the above conditions (1) and (2) as follows: Let $\mathfrak{M}=\mathfrak{M}^{(0)}$ be a sys-

Received 26 July, 1952.

1) Nagoya Math. Journ. 1 (1950), pp. 63-70.

2) Prof. I. S. Cohen (Massachusetts In stitute of Technology, U.S.A.) pointed out the error of the proof of Proposition 2. I am grateful to him for his kind communication. 
tem of representatives of a $p$-basis of $M R / \mathfrak{m}$. Let $\mathfrak{M}^{(n)}$ be, when $\mathbb{M}^{(n-1)}$ is already given, a set such that (1) for every element of $\mathfrak{M}^{(n-1)}, \mathfrak{M}^{(n)}$ contains one and only one $p$-th root of it and (2) $\mathfrak{D}^{(n)}$ consists merely of $p$-th roots of elements of $\mathfrak{M}^{(n-1)}$. Set $R^{(n)}=R\left[\mathfrak{M}^{(n)}\right]$.

Let $S$ be the union of all $R^{(n)}$ and let $\bar{S}$ be its completion. Then we see easily that the multiplicative representative of an arbitrary element of $\mathfrak{D}$ is itself. Let $R_{0}$ be the absolutely unramified local ring which is generated by multiplicative representatives for $R / \mathrm{m}$. Now, for our purpose, it is sufficient to prove the following.

Lemma. For every element $a$ of $R_{0}$, there exists an element $a_{n}$ of $R$ such that $a \equiv a_{n}\left(\bmod . \mathrm{m}^{n} \bar{S}\right)$.

Proof. For $n=1$, our assertion is evident. We assume that this is true for $n=r$ and we prove the case $n=r+1$. Since $R_{0} /(p)=R / \mathrm{m}=\left(R_{0} /(p)\right)^{p^{r}}(M)$, we can find an element $c_{1}=\sum_{i} b_{i}^{p^{r}} m_{i}$ (where $b_{i} \in R_{0}$ and $m_{i}$ is a monomial on elements of $\mathfrak{M})$ such that $a=c_{1}+p c_{2}\left(c_{2} \in R_{0}\right)$. Let $b_{i}^{\prime}$ be an element of $R$ such that $b_{i} \equiv b_{i}^{\prime}(\bmod . m \bar{S})$ and let $c^{\prime}$ be an element of $R$ such that $c \equiv c^{\prime}\left(\bmod . m^{r} \bar{S}\right)$. Then $a_{n}=\sum_{i} b_{i}^{\prime p^{r}} m_{i}+p c^{\prime}$ is a required element.

Proof of the Corollary to Proposition 3.

As is obvious, we have only to treat the case when $\bar{R}_{0}$ is of characteristic 0 and $p \neq 0$. Let $B$ be a complete valuation ring (of characteristic 0 ) such that $B /(p)=\bar{R}_{0} /(p)$.

(1) When $\bar{R}_{0} /(p)$ is perfect:

Let $\left\{\bar{y}_{\lambda}\right\}$ be a transcendental basis for $\bar{R}_{0} /(p)$ over the prime field. Then we can find its multiplicative representative systems $\left\{y_{\nu}\right\},\left\{z_{\lambda}\right\}$ in $\bar{R}_{0}$ and $B$. Then we can identify $z_{\lambda}$ with $y_{\lambda}$. The same holds for $\left\{\bar{y}_{\lambda}^{p^{-n}}\right\}$ and the similar identification allows the above identification of $y_{\lambda}$ and $z_{\lambda}$. Therefore we may consider that $\bar{R}_{0}$ and $B$ contains the same complete valuation ring $B_{1}$ such that its residue field is the least perfect field containing $\left\{\bar{y}_{\lambda}\right\}_{\text {. Since }} \bar{R}_{0} /(p)$ is separably algebraic over $B_{1} /(p)$ and since $B$ is complete, we see that $B$ and $\bar{R}_{0}$ are isomorphic over $B_{1}$.

(2) General case :

Considering $\bar{R}_{0}$ as $R$ in the above proof of Proposition 2, we construct the valuation ring $\bar{S}$. Let $K$ be the largest perfect subfield of $\bar{R}_{0} /(p)$. Then using multiplicative representatives for $K$ in $\bar{R}_{0}$ and $B$, we see that $\bar{R}_{0}$ and $B$ contain, respectively, complete valuation rings $B_{1}$ and $B_{1}^{\prime}$ with the same residue field $K$. Then by (1), we may identify $B_{1}^{\prime}$ with $B_{1}$. Further, we may assume without loss of generality that $\mathfrak{M}$ ( $=$ a system of representatives of $p$-basis in $\bar{R}_{0}$ ) is also contained in $B$. Then our assertion follows immediately by our above 
proof of Proposition 2.

\section{Errata :}

p. $63, l .21$ and p. 64, l.27; For "form" read "forms", p. 66, Proposition 2; For "with maximal ideal" read "with maximal ideal m", p. 69, Proposition 7; For "With these conditions" read "If these conditions".

Mathematical Institute, Nagoya University 